\title{
79. APORTACIONES A LA FLORA DE ANDALUCÍA
}

\author{
Manuel BECERRA, Juan A. GARCÍA ROJAS, Andrés V. PÉREZ-LATORRE, \\ Baltasar CABEZUDO y Teresa NAVARRO
}

New data on the Andalusian flora.

Palabras clave. Papaver, Erica, Convolvulus, Teucrium, flora, corología, Andalucía, España.

Key words. Papaver, Erica, Convolvulus, Teucrium, flora, chorology, Andalusia, Spain.

\section{Papaver rupifragum Boiss. \& Reuter}

MÁLAGA: Benaoján. Parque Natural de Grazalema. Sierra del Palo, 1310 m. s. m., 6.VI.2001, Becerra, Cabezudo, D. Navas y Pérez-Latorre. MGC 48000.

Especie endémica de Andalucía y Marruecos. En Andalucía se localizaba exclusivamente en la provincia de Cádiz (P. N. de Grazalema) (Silvestre, 1999; Díaz González, 1986). Es una especie protegida considerada En Peligro de Extinción por la Junta de Andalucía y En Peligro según criterios UICN (Silvestre 1999). La localización de esta población en la provincia de Málaga puede favorecer los planes de recuperación de esta especie en el parque natural, siempre que se proteja específicamente la zona donde se desarrolla. El número de individuos asciende a 573 ocupando una superficie de $230 \mathrm{~m}^{2}$ ( 2.5 indv. por $\left.\mathrm{m}^{2}\right)$, todos se encontraban en flor o fruto, observándose una fuerte predación de ambas estructuras de reproducción. Hemos calculado la edad de los individuos en función del número de escapos florales por pie con el siguiente resultado: $16.75 \%$ ( 1 escapo), $30.9 \%$ (2 escapos), $27.74 \%$ (3 escapos), $21.73 \%$ (4 escapos) y $11.88 \%$ (5 o mas escapos). Las poblaciones malagueñas de $P$. rupifragum se desarrollan en lapiaces hipercarstificados con canchales calizos estabilizados en el piso bioclimático mesomediterráneo superior con ombroclima hiperhúmedo; la vegetación potencial corresponde a los encinares con quejigos de Paeonio-Quercetum rotundifoliae quercetosum fagineae. Un listado realizado en la localidad, con vegetación de contacto de Festucion scariosae con Helleborus phoetidus, arroja las siguientes especies: Acinos alpinus subsp. meridionalis, Cerastium boissieri, Dactylis hispanica, Linaria tristis, Asplenium ceterach y
Phlomis composita, lo que permite incluir esta comunidad en Phagnalo-Rumicetalia indurati.

Erica andevalensis Cabezudo \& Rivera

SEVILLA: Aznalcóllar. Corredor del Guadiamar. Rio Crispinejo. 120 m. s. m., 20.VI.2001, Cabezudo, Pérez-Latorre y P. Navas. MGC 48632.

Edafoendemismo andaluz indicado, hasta el momento, solo en la provincia de Huelva (Bayer, 1993; Aparicio, 1999 y López González, 2001). Con motivo de los trabajos que estamos realizando para la recuperación de la vegetación en el área del Corredor Verde del Guadiamar (Proyecto Junta de Andalucía), hemos localizado varias poblaciones en la cuenca sevillana del río Crispinejo, poblaciones que pueden favorecer la recuperación natural de esta especie en la cuenca del río Guadiamar. Es una especie En Peligro de Extinción (Junta de Andalucía) y Vulnerable según criterios UICN. E. andevalensis aparece en juncal-brezales edafohigrófilos metalícolas, ya descritos por Cabezudo et al. (1989) como Junco rugosi-Ericetum andevalensis, aunque ésta localidad presenta especies como Erica scoparia y Erica lusitanica que indican un biotopo con mayor humedad temporal.

\section{Convolvulus boissieri Steudel}

GRANADA: Alhama de Granada. Parque Natural de Sierras Tejeda y Almijara. Puerto del Lobo. 1850 m. s. m. 12.VII.2001. Cabezudo, P. Navas, Y. Gil y Pérez Latorre. MGC 48055.

Convolvulus boissieri se creía ausente del sector Almijaro-Granatense (sensu Nieto et al., 1991) en su parte suroccidental: Sierras de Tejeda, Almijara, Alhama, siendo sin embargo muy abundante Pterocephalus spathulatus, una especie 
ecomorfológicamente muy similar (Nieto et al., 1989). Sin embargo hemos localizado una pequeña población en Sierra Tejeda en su vertiente granadina. C. boissieri se desarrolla aquí en un biotopo que no es el clásico de la especie (litosuelos arenosos de dolomías diaclasadas) sino que aparece sobre mármoles masivos en espolones muy expuestos con litosuelos pavimentados, en el piso bioclimático supramediterráneo superior (oromediterráneo inferior topográfico). La comunidad en que habita C. boissieri es incluible en Minuartio-Poion ligulatae (Festuco-Poetalia, Festuco-Ononidetea) y se acerca al Seselido-Festucetum hystricis arenarietosum erinacei (Pérez Latorre et al., 1998) en su variante almijarense con Armeria filicaulis. Un inventario levantado en la localidad arroja la siguiente composición: altitud 1850 m, orientación norte, cobertura $60 \%$, área $5 \mathrm{~m}^{2}$, Convolvulus boissieri 3, Erodium cheilanthifolium 2, Erinacea anthyllis 1, Koeleria vallesiana 2, Vella spinosa 1, Armeria filicaulis + , Arenaria erinacea 2, Sedum acre 1, Helianthemum appeninum 1, Brassica repanda subsp. latisiliqua + , Anthyllis tejedensis + , Asperula hirsuta + , Salvia lavandulifolia + . La vegetación climácica en el biotopo donde se desarrolla la comunidad de C. boissieri corresponde a sabinares edafoxerófilos permanentes de RhamnoJuniperetum phoeniceae, aunque el dominio climatófilo en los alrededores sobre suelos zonales es de Daphno-Aceretum granatensis, en tránsito ya a formaciones oromediterráneas de Juniperus communis. Esta localidad rellena el espacio corológico en blanco existente entre las poblaciones señaladas por Pérez Latorre et al. (1998) en la Serranía de Ronda y las ya conocidas de la orla dolomítica de Sierra Nevada (Pérez Raya et al., 1990).

\section{Teucrium bracteatum Desf.}

CÁDIZ: Tarifa. Junto al mirador del Estrecho. 240 m. s. m., 9.VI.2001, J. A. García Rojas. MGC 49242.

Teucrium bracteatum Desf., especie incluida en la sección Stachyobotrys Bethn., es un hemicriptófito perenne deciduo de invierno con floración estival que vive principalmente en colinas pedregosas y soleadas. Constituye junto con $T$. collincola Greuter \& Burdet, endemismo marroquí, la única representación de esta sección en el Mediterráneo occidental (Navarro \& El Oualidi,
2000). T. bracteatum se distribuye por Argelia, Marruecos y en la Península Ibérica. La primera cita publicada (MGC 36236) de la presencia de esta especie en el norte del Mediterráneo, es reciente y corresponde a la Sierra Prieta de Casarabonela en la provincia de Málaga (Navarro \& Cabezudo, 1997). Sin embargo, la referencia más antigua en la que se cita $T$. bracteatum en la Península Ibérica se debe a Ball (1878: 630). Este autor menciona la localidad de Tarifa dentro de su área de distribución, especificando que se trata de una localidad de dónde no existían recolecciones recientes conocidas. La presente cita corológica confirma la antigua cita de Ball (op. cit.) y la recogida en la Flora Calpensis (Wolley, 1949). El material de T. bracteatum de Tarifa, permite ampliar la distribución de esta especie en la Peninsula Ibérica a la provincia de Cádiz. Flora Calpensis es una obra manuscrita, editada por la British Crown Colony de Gibraltar y depositada en el Consulado Americano de Gibraltar el 30 de abril de 1949. Se basa sobre material recolectado por el coronel americano D. Wolley depositado en 1949 en el herbario del Gibraltar Museum Colonel Wolley Dod. En esta obra manuscrita se indica que la especie había sido recolectada por primera vez en la península Ibérica "entre Carnero y Pelayo (Tarifa)". La imposibilidad de confirmación de esta cita sobre material de herbario original de Wolley, hace del pliego (MGC 49242) el testigo que confirma la presencia de $T$. bracteatum en Tarifa. La presencia de esta especie en el norte del Mediterráneo (suroeste de Andalucía) puede considerarse como rara, pues ambas poblaciones (Casarabonela y Tarifa) cuentan con escasos individuos y de difícil localización. Además las dos poblaciones habitan en comunidades bastante diferentes, ya que en la Sierra Prieta $T$. bracteatum ocupa suelos pedregosos sobre calizas y dolomías (Andryalo-Crambion filiformis) y en Tarifa aparentemente se encuentra en taludes silíceos con especies de Sysimbrietalia officinalis.

\section{BIBLIOGRAFÍA}

APARICIO, A. -1999- Erica andevalensis, en Blanca, G., B. Cabezudo, J, E. Hernández Bermejo, C. M. Herrera, J. Molero Mesa, J. Muñoz y B. Valdés. Libro Rojo de la flora 
silvestre amenazada de Andalucía. I. Especies En Peligro de Extinción: 119-122. Junta de Andalucía.

BALL, J. -1878- Spicilegium Florae Maroccanae. J. Linn. Soc. 16: 281-772 (1877-1878).

BAYER, E. -1993- Erica, en S. Castroviejo et al. (Eds.) Flora Iberica IV: 491. CSIC. Madrid.

CABEZUDO, B., J. M. NIETO CALDERA y A. V. PÉREZ LATORRE -1989- Junco rugosiEricetum andevalensis ass. nova. Acta Bot. Malacitana 14:294-296.

DÍAZ GONZÁLEZ, T. E. -1986- Papaver L. en S. Castroviejo et al. (Eds.) Flora Iberica I: 407417. CSIC. Madrid.

LÓPEZ GONZÁLEZ, G. -2001- Los arboles y arbustos de la Península Ibérica e Islas baleares. Madrid.

NAVARRO, T. y B. CABEZUDO -1997- Dos novedades del género Teucrium sección Stachyobotrys Benth. y sección Scorodonia (Hill) Schreb. subsección Scorodonia Kästner (Lamiaceae) para la flora europea. Acta Bot. Malacitana 22: 249-250.

NAVARRO, T. \& J. EL OUALIDI -2000- Synopsis of Teucrium L. (Labiatae) in the Mediterranean region and surrounding areas. Flora Medit. 10: 349-363.

NIETO CALDERA, J. M., A. V. PÉREZ LATORRE y B. CABEZUDO -1991- Biogeografía y series de vegetación de la provincia de Málaga (España). Acta Bot. Malacitana 16(2):417-436.

NIETO CALDERA, J. M., B. CABEZUDO y M. M. TRIGO PÉREZ -1989- Series de vegetación edafófilas de las Sierras Tejeda y Almijara (Málaga, Granada, España). Acta Bot. Malacitana 14:161-170

PÉREZ LATORRE, A. V. y B. CABEZUDO -1998Datos sobre la flora y vegetación de la Serranía de Ronda. Acta Bot. Malacitana 23:149-191.

PÉREZ RAYA, F., J. M. LÓPEZ NIETO, J. MOLERO MESA y F. VALLE -1990Vegetación de Sierra Nevada. Ayuntamiento de Granada y Universidad de Granada.

SILVESTRE, S. -1999-. Papaver rupifragum, en Blanca, G., B. Cabezudo, J, E. Hernández Bermejo, C. M. Herrera, J. Molero Mesa, J. Muñoz y B. Valdés. Libro Rojo de la flora silvestre amenazada de Andalucía. I. Especies En Peligro de Extinción: 219-223. Junta de Andalucía.
WOLLEY, A. H. -1949- Flora Calpensis. A List of Plants Recorded from Gibraltar and the Campo District of Spain. Consulado Americano. Gibraltar.

Aceptado para su publicación en octubre de 2001

Dirección de los autores. Departamento de Biología Vegetal. Facultad de Ciencias. Universidad de Málaga. Campus de Teatinos. 29071, Málaga. 